\title{
The Exponentiated Generalized Lindley Distribution
}

\author{
J. A. Rodrigues ${ }^{1^{*}}$, A. C. Percontini ${ }^{2}$ and G. G. Hamedani ${ }^{3}$ \\ ${ }^{1}$ Department of Education, Federal Institute of Bahia, Feira de Santana, 44.096-486, Brasil. \\ ${ }^{2}$ Department of Mathematics, State University of Feira de Santana, Feira de Santana, 44.031-460, \\ Brasil. \\ ${ }^{3}$ Department of Mathematics, Statistics and Computer Science, Marquette University, Milwaukee,
} 53201-1881, United States of America.

Authors' contributions

This work was carried out in collaboration between all authors. All authors read and approved the final manuscript..

Article Information

DOI: $10.9734 /$ ARJOM/2017/33206 Editor(s):

(1) Xiao-Jun Yang, Department of Mathematics and Mechanics, China University of Mining and Technology, Xuzhou 221008, China.

(2) Jaya Bishwal, Department of Mathematics and Statistics, University of North Carolina at Charlotte, USA.

(3) Hamidah Ibrahim, Department of Computer Science, Universiti Putra Malaysia, Malaysia. Reviewers:

(1) Septiadi Padmadisastra, Uni. Padjadjaran, Indonesia. (2) Thomas L. Toulias, Technological Educational Institute of Athens, Greece.

(3) Min Wang, Michigan Technological University, USA.

(4) G. G. Hamedani, Marquette University, USA.

(5) Anonymous, Miami Dade College, USA.

Complete Peer review History: http://www.sciencedomain.org/review-history/20136

Original Research Article

Received: $9^{\text {th }}$ May 2016

Accepted: $14^{\text {th }}$ June 2017

Published: $20^{\text {th }}$ July 2017

\begin{abstract}
A new distribution called the exponentiated generalized Lindley is proposed and studied. This distribution includes as special cases the Lindley and exponentiated Lindley distributions. We study the main properties of this distribution, with special emphasis on its moments and some characteristics related to reliability studies. The estimation of the model parameters using the methods of moments and maximum likelihood is also discussed. The flexibility of this distribution is illustrated via an application to a real data set.
\end{abstract}

*Corresponding author: E-mail: jailsondearaujo@yahoo.com.br; 
Keywords: Akaike information criterion; characterizations; Hazard function; lindley distribution; maximum likelihood estimators; moments.

2010 Mathematics Subject Classification: 60E05, 62H10.

\section{Introduction}

The probability distributions have been extensively used to describe real world phenomena. Due to the usefulness of probability distributions, their theory is widely studied and new distributions are developed. For example, [1] proposed the Weibull-Pareto distribution. [2] introduced the betaDagum distribution. [3] proposed the Mc-Dagum distribution and discussed its various properties. [4] introduced and studied the exponentiated Lindley distribution. [5] defined a five-parameter beta Burr XII distribution and discussed its various properties. [6] introduced the Kumaraswamy generalized gamma distribution. [7] studied the gamma-exponentiated Weibull distribution and [8] studied the beta modified Weibull distribution.

The Lindley distribution is a popular life time probability distribution that has been used for modeling in actuarial sciences, engineering and biological studies.

In this work, we propose a new distribution that extends the Lindley distribution. Some of the main structural properties of this distribution are derived. The estimation of parameters using the methods of moments and maximum likelihood is also discussed. The flexibility of this distribution is illustrated via an application to a real data set.

The new distribution will serve as an alternative model to other Models available in the literature for modeling positive real data in many areas.

The article is organized as follows. In Section 2, the exponentiated generalized Lindley distribution is defined and some special sub-models are discussed. The moments and moment generating function are derived in Section 3. Characterizations of the new model are presented in Section 4. The estimation of the model parameters using the methods of moments and maximum likelihood is discussed in Section 5. Finally, in Section 6 an application to a real data set is reported.

\section{The Model}

[9] introduced a one-parameter distribution, known as Lindley distribution, given by its probability density function (PDF)

$$
g(x ; \lambda)=\frac{\lambda^{2}}{\lambda+1}(1+x) e^{-\lambda x},
$$

for $x>0$ and $\lambda>0$. The corresponding cumulative distribution function (CDF) is

$$
G(x ; \lambda)=1-\left(1+\frac{\lambda x}{\lambda+1}\right) e^{-\lambda x}
$$

Let $G(x)$ be the CDF of any random variable $X$. The CDF of a generalized class of distributions, defined by [10], is given by

$$
F(x ; \alpha, \beta)=\left\{1-[1-G(x)]^{\alpha}\right\}^{\beta},
$$


where $\alpha>0$ and $\beta>0$ are two additional shape parameters. The corresponding PDF for (2.3) is given by

$$
f(x ; \alpha, \beta)=\alpha \beta g(x)[1-G(x)]^{\alpha-1}\left\{1-[1-G(x)]^{\alpha}\right\}^{\beta-1} .
$$

Replacing (2.2) in (2.3), we obtain a new distribution, called exponentiated generalized Lindley (EGL), with CDF given by

$$
F(x ; \alpha, \beta, \lambda)=\left[1-\left(\left(1+\frac{\lambda x}{\lambda+1}\right) e^{-\lambda x}\right)^{\alpha}\right]^{\beta}
$$

The PDF corresponding to $F(x ; \alpha, \beta, \lambda)$ is

$$
f(x ; \alpha, \beta, \lambda)=\frac{\alpha \beta \lambda^{2}}{\lambda+1}(1+x)\left(1+\frac{\lambda x}{\lambda+1}\right)^{\alpha-1}\left[1-\left(\left(1+\frac{\lambda x}{\lambda+1}\right) e^{-\lambda x}\right)^{\alpha}\right]^{\beta-1} e^{-\alpha \lambda x}
$$

Fig. 1 shows the graphs of PDF of EGL distribution for various values of the parameters $\alpha, \beta$ and $\lambda$.

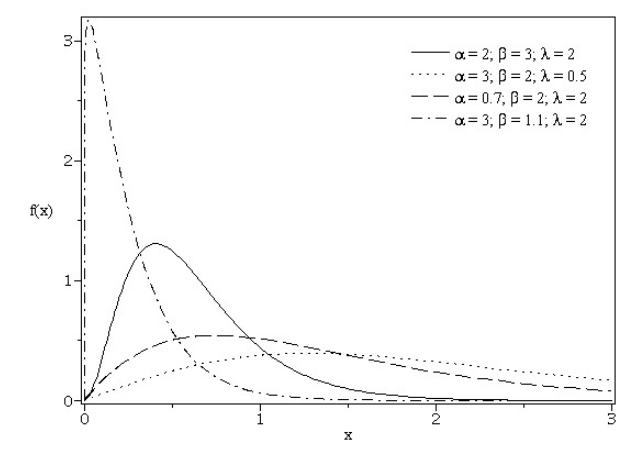

Fig. 1. Graphs of the PDF of the EGL distribution for different values of the parameters

\section{$2.1 \quad$ Sub-models}

Sub-models of EGL distribution for selected values of the parameters are presented in this subsection.

1. When $\alpha=1$, the EGL distribution is the exponentiated Lindley (EL) distribution, [4], with the density given by

$$
f(x ; \beta, \lambda)=\frac{\beta \lambda^{2}}{\lambda+1}(1+x)\left[1-\left(1+\frac{\lambda x}{\lambda+1}\right) e^{-\lambda x}\right]^{\beta-1} e^{-\lambda x}
$$

2. If $\beta=1$, we have the generalized Lindley distribution with the density given by

$$
f(x ; \alpha, \lambda)=\frac{\alpha \lambda^{2}}{\lambda+1}(1+x)\left(1+\frac{\lambda x}{\lambda+1}\right)^{\alpha-1} e^{-\alpha \lambda x} ;
$$




\section{Properties of the Model}

\subsection{Expansions for the cumulative and density functions}

For any real non-integer $\beta>0$, [11] defined the power series

$$
(1-z)^{\beta}=\beta \sum_{j=0}^{\infty} \frac{(-1)^{j} \Gamma(\beta) z^{j}}{\Gamma(\beta-j+1) j !},
$$

where $|z|<1$. Using the series representation (3.1) in Equation 2.5, we can write

$$
F(x ; \alpha, \beta, \lambda)=\beta \sum_{j=0}^{\infty} \frac{(-1)^{j} \Gamma(\beta)}{\Gamma(\beta-j+1) j !}\left(\frac{\lambda+1+\lambda x}{\lambda+1} \cdot e^{-\lambda x}\right)^{\alpha j} .
$$

If $\alpha>0$ is an integer and $\beta$ is a real non-integer, we can write (3.2), through the known Binomial Theorem, as

$$
F(x ; \alpha, \beta, \lambda)=\beta \sum_{j=0}^{\infty} \sum_{k=0}^{\alpha j} \frac{(-1)^{j} \Gamma(\beta)(\alpha j) !}{\Gamma(\beta-j+1)(\alpha j-k) ! j ! k !}\left(\frac{\lambda}{\lambda+1}\right)^{k} x^{k} e^{-\alpha \lambda j x} .
$$

Using again the series (3.1), we can express the PDF of EGL distribution as

$$
f(x ; \alpha, \beta, \lambda)=\frac{\alpha \beta \lambda^{2}}{\lambda+1}(x+1) \sum_{j=0}^{\infty} \frac{(-1)^{j} \Gamma(\beta)}{\Gamma(\beta-j) j !}\left(\frac{\lambda+1+\lambda x}{\lambda+1}\right)^{\alpha(j+1)-1} e^{-\alpha \lambda(j+1) x} .
$$

If $\alpha$ is an integer and $\beta$ is a real non-integer, we can write (3.4), also through the known Binomial Theorem, as

$$
f(x ; \alpha, \beta, \lambda)=\frac{\alpha \beta \lambda^{2}}{\lambda+1}(x+1) \sum_{j=0}^{\infty} \sum_{k=0}^{\alpha(j+1)-1} \frac{(-1)^{j} \Gamma(\beta)[\alpha(j+1)-1] !}{\Gamma(\beta-j)[\alpha(j+1)-k-1] ! j ! k !}\left(\frac{\lambda}{\lambda+1}\right)^{k} x^{k} e^{-\alpha \lambda(j+1) x} .
$$

For $\beta$ integer, the index $j$ in the previous sums stops at $\beta$.

\subsection{Moments}

Lemma 1. (Equation (2.3.6.9), [12]). If $0<\operatorname{Re}(a), 0<\operatorname{Re}(p)$ and $|\arg (z)|<\pi$ then

$$
\int_{0}^{\infty} x^{a-1}(x+z)^{-\rho} \exp (-p x) d x=\Gamma(a) z^{a-\rho} \Psi(a, a+1-\rho ; p z),
$$

where $\Psi(a, b ; x)$ is the Gordon function defined by

$$
\Psi(a, b ; x)=\frac{\Gamma(b-1)}{\Gamma(a)} x^{1-b} \sum_{k=0}^{\infty} \frac{(a-b+1)_{k} x^{k}}{(2-b)_{k} k !}+\frac{\Gamma(1-b)}{\Gamma(a-b+1)} \sum_{k=0}^{\infty} \frac{(a)_{k} x^{k}}{(b)_{k} k !}
$$

and $(x)_{j}=(x)(x+1) \ldots(x+j-1)$ denotes the Pochhammer symbol.

Lemma 2. The $n$-th raw moment of the EGL distribution, as in (3.4), for $\beta>0$ real non-integer, is given by

$$
\begin{aligned}
E\left(X^{n}\right) & =\alpha \beta \lambda n !\left(\frac{\lambda+1}{\lambda}\right)^{n} \sum_{j=0}^{\infty} \frac{(-1)^{j} \Gamma(\beta)}{\Gamma(\beta-j) j !}\{\Psi(n+1, n+\alpha(j+1)+1 ; \alpha(j+1)(\lambda+1)) \\
& +[(n+1)(\lambda+1) / \lambda] \Psi(n+2, n+\alpha(j+1)+2 ; \alpha(j+1)(\lambda+1))\} .
\end{aligned}
$$

If $\beta>0$ is an integer, the index $j$ stops at $\beta$. 
Proof. The $n$-th raw moment of the EGL distribution, as in (3.4), is given by

$$
\begin{aligned}
E\left(X^{n}\right) & =\int_{0}^{\infty} x^{n} f(x ; \alpha, \beta, \lambda) d x \\
& =\frac{\alpha \beta \lambda^{2}}{\lambda+1} \sum_{j=0}^{\infty} \frac{(-1)^{j} \Gamma(\beta)}{\Gamma(\beta-j) j !} \int_{0}^{\infty} x^{n}(x+1)\left(\frac{\lambda+1+\lambda x}{\lambda+1}\right)^{\alpha(j+1)-1} e^{-\alpha \lambda(j+1) x} d x
\end{aligned}
$$

It follows from Lemma 1 that the $n$-th raw moment of the EGL distribution is given by

$$
\begin{aligned}
E\left(X^{n}\right) & =\alpha \beta \lambda n !\left(\frac{\lambda+1}{\lambda}\right)^{n} \sum_{j=0}^{\infty} \frac{(-1)^{j} \Gamma(\beta)}{\Gamma(\beta-j) j !}\{\Psi(n+1, n+\alpha(j+1)+1 ; \alpha(j+1)(\lambda+1)) \\
& +[(n+1)(\lambda+1) / \lambda] \Psi(n+2, n+\alpha(j+1)+2 ; \alpha(j+1)(\lambda+1))\} .
\end{aligned}
$$

\subsection{Moment generating function}

Lemma 3. The moment generating function of $E G L$ distribution is given by

$$
\begin{aligned}
M(t) & =\alpha \beta \lambda \sum_{j=0}^{\infty} \frac{(-1)^{j} \Gamma(\beta)}{\Gamma(\beta-j) j !}\{\Psi(1, \alpha(j+1)+1 ;[\alpha \lambda(j+1)-t](\lambda+1) / \lambda) \\
& +[(\lambda+1) / \lambda] \Psi(2, \alpha(j+1)+2 ;[\alpha \lambda(j+1)-t](\lambda+1) / \lambda)\}
\end{aligned}
$$

where $t<\alpha \lambda$. The corresponding characteristic function is

$$
\begin{aligned}
\phi(t) & =\alpha \beta \lambda \sum_{j=0}^{\infty} \frac{(-1)^{j} \Gamma(\beta)}{\Gamma(\beta-j) j !}\{\Psi(1, \alpha(j+1)+1 ;[\alpha \lambda(j+1)-i t](\lambda+1) / \lambda) \\
& +[(\lambda+1) / \lambda] \Psi(2, \alpha(j+1)+2 ;[\alpha \lambda(j+1)-i t](\lambda+1) / \lambda)\} .
\end{aligned}
$$

where $i=\sqrt{-1}$.

Proof. The moment generating function of EGL distribution is given by

$$
\begin{aligned}
M(t) & =\int_{0}^{\infty} e^{t x} f(x ; \alpha, \beta, \lambda) d x \\
& =\frac{\alpha \beta \lambda^{2}}{\lambda+1} \sum_{j=0}^{\infty} \frac{(-1)^{j} \Gamma(\beta)}{\Gamma(\beta-j) j !} \int_{0}^{\infty} e^{t x}(x+1)\left(\frac{\lambda+1+\lambda x}{\lambda+1}\right)^{\alpha(j+1)-1} e^{-\alpha \lambda(j+1) x} d x \\
& =\frac{\alpha \beta \lambda^{2}}{\lambda+1} \sum_{j=0}^{\infty} \frac{(-1)^{j} \Gamma(\beta)}{\Gamma(\beta-j) j !} \int_{0}^{\infty}(x+1)\left(\frac{\lambda+1+\lambda x}{\lambda+1}\right)^{\alpha(j+1)-1} e^{-[\alpha \lambda(j+1)-t] x} d x
\end{aligned}
$$

The application of Lemma 1 shows that (3.13) can be rewritten as

$$
\begin{aligned}
M(t) & =\alpha \beta \lambda \sum_{j=0}^{\infty} \frac{(-1)^{j} \Gamma(\beta)}{\Gamma(\beta-j) j !}\{\Psi(1, \alpha(j+1)+1 ;[\alpha \lambda(j+1)-t](\lambda+1) / \lambda) \\
& +\quad[(\lambda+1) / \lambda] \Psi(2, \alpha(j+1)+2 ;[\alpha \lambda(j+1)-t](\lambda+1) / \lambda)\} .
\end{aligned}
$$




\subsection{Order statistics}

Suppose $X_{1}, X_{1}, \ldots, X_{n}$ is a random sample from EGL distribution. Let $X_{1: n}<X_{2: n}<\ldots<X_{n: n}$ denote the corresponding order statistics. From [13], the PDF and CDF of the $r$ th order statistic, say $Y=X_{r: n}$, are given by

$$
\begin{aligned}
f_{Y}(y) & =\frac{n !}{(r-1) !(n-r) !} F^{r-1}(y)[1-F(y)]^{n-r} f(y) \\
& =\frac{n !}{(r-1) !(n-r) !} \sum_{l=0}^{n-r}\left(\begin{array}{c}
n-r \\
l
\end{array}\right)(-1)^{l} F^{l+r-1}(y) f(y)
\end{aligned}
$$

and

$$
\begin{aligned}
F_{Y}(y) & =\sum_{j=r}^{n}\left(\begin{array}{c}
n \\
j
\end{array}\right) F^{j}(y)[1-F(y)]^{n-j} \\
& =\sum_{j=r}^{n} \sum_{l=0}^{n-j}\left(\begin{array}{c}
n \\
j
\end{array}\right)\left(\begin{array}{c}
n-j \\
l
\end{array}\right)(-1)^{l} F^{j+l}(y),
\end{aligned}
$$

where $f(\cdot)$ and $F(\cdot)$ are the PDF and CDF of the EGL distribution, respectively. It follows from Equations 2.5 and 2.6 that

$$
\begin{aligned}
f_{Y}(y) & =\frac{\alpha \beta \lambda^{2}(1+y) n !}{(\lambda+1)(r-1) !(n-r) !}\left(\frac{\lambda+1+\lambda y}{\lambda+1}\right)^{\alpha-1} \sum_{l=0}^{n-r}\left(\begin{array}{c}
n-r \\
l
\end{array}\right)(-1)^{l} e^{-\alpha \lambda y} \\
& \times\left[1-\left(\frac{\lambda+1+\lambda y}{\lambda+1} e^{-\lambda y}\right)^{\alpha}\right]^{\beta(l+r)-1}
\end{aligned}
$$

and

$$
F_{Y}(y)=\sum_{j=r}^{n} \sum_{l=0}^{n-j}\left(\begin{array}{c}
n \\
j
\end{array}\right)\left(\begin{array}{c}
n-j \\
l
\end{array}\right)(-1)^{l}\left[1-\left(\frac{\lambda+1+\lambda y}{\lambda+1} e^{-\lambda y}\right)^{\alpha}\right]^{\beta(j+l)}
$$

\section{Characterizations of Model}

Characterizations of distributions is an important research area which has recently attracted the attention of many researchers. This section deals with various characterizations of the EGL distribution. These characterizations are based on: $(i)$ a simple relationship between two truncated moments; ( $i$ i) the hazard function; (iii) the reverse (or reversed) hazard function and (iv) conditional expectation of a function of the random variable. It should be mentioned that for characterization (i) the CDF is not required to have a closed form.

We present our characterizations $(i)-(i v)$ in four subsections.

\subsection{Characterizations based on truncated moments}

In this subsection we present characterizations of EGL distribution in terms of a simple relationship between two truncated moments. The first characterization result employs a theorem due to [14], see Theorem 2.1.1 below. Note that the result holds also when the interval $H$ is not closed. Moreover, as mentioned above, it could be also applied when the CDF $F$ does not have a closed form. As shown in [15], this characterization is stable in the sense of weak convergence. 
Theorem 4.1 Let $(\Omega, \mathcal{F}, \mathbf{P})$ be a given probability space and let $H=[d, e]$ be an interval for some $d<e(d=-\infty, e=\infty$ might as well be allowed). Let $X: \Omega \rightarrow H$ be a continuous random variable with the distribution function $F$ and let $g$ and $h$ be two real functions defined on $H$ such that

$$
\mathbf{E}[g(X) \mid X \geq x]=\mathbf{E}[h(X) \mid X \geq x] \xi(x), \quad x \in H,
$$

is defined with some real function $\eta$. Assume that $g, h \in C^{1}(H), \xi \in C^{2}(H)$ and $F$ is twice continuously differentiable and strictly monotone function on the set $H$. Finally, assume that the equation $\xi h=g$ has no real solution in the interior of $H$. Then $F$ is uniquely determined by the functions $g, h$ and $\xi$, particularly

$$
F(x)=\int_{a}^{x} C\left|\frac{\xi^{\prime}(u)}{\xi(u) h(u)-g(u)}\right| \exp (-s(u)) d u,
$$

where the function $s$ is a solution of the differential equation $s^{\prime}=\frac{\xi^{\prime} h}{\xi h-g}$ and $C$ is the normalization constant, such that $\int_{H} d F=1$.

Proposition 4.1 Let $X: \Omega \rightarrow(0, \infty)$ be a continuous random variable and let , $h(x)=$ $\left[1-\left(1+\frac{\lambda x}{\lambda+1}\right)^{\alpha} e^{-\alpha \lambda x}\right]^{1-\beta}$ and $g(x)=h(x)\left(1+\frac{\lambda x}{\lambda+1}\right) e^{-\lambda x}$ for $x>0$. The random variable $X$ has PDF (2.6) if and only if the function $\xi$ defined in Theorem 4.1 has the form

$$
\xi(x)=\frac{\alpha}{\alpha+1}\left(1+\frac{\lambda x}{\lambda+1}\right) e^{-\lambda x}, \quad x>0 .
$$

Proof. Let $X$ be a random variable with PDF (2.6), then

$$
(1-F(x)) E[h(x) \mid X \geq x]=\beta\left(1+\frac{\lambda x}{\lambda+1}\right)^{\alpha} e^{-\alpha \lambda x}, \quad x>0,
$$

and

$$
(1-F(x)) E[g(x) \mid X \geq x]=\frac{\alpha}{\alpha+1}\left(1+\frac{\lambda x}{\lambda+1}\right)^{\alpha} e^{-\lambda(\alpha+1) x}, \quad x>0,
$$

and finally

$$
\xi(x) h(x)-g(x)=h(x)\left[-\frac{1}{\alpha+1}\left(1+\frac{\lambda x}{\lambda+1}\right) e^{-\lambda x}\right]<0 \quad \text { for } x>0 .
$$

Conversely, if $\xi$ is given as above, then

and hence

$$
s^{\prime}(x)=\frac{\xi^{\prime}(x) h(x)}{\xi(x) h(x)-g(x)}=\frac{\alpha \lambda^{2}(1+x)}{1+\lambda(1+x)} \quad x>0,
$$

$$
s(x)=\alpha \lambda x-\alpha \log (1+\lambda(1+x)), \quad x>0 .
$$

Now, in view of Theorem A.1.1, $X$ has density (2.6). 
Corollary 4.1 Let $X: \Omega \rightarrow(0, \infty)$ be a continuous random variable and let $h(x)$ be as in Proposition A.1.1. The PDF of $X$ is (2.6) if and only if there exist functions $g$ and $\xi$ defined in Theorem 4.1 satisfying the differential equation

$$
\frac{\xi^{\prime}(x) h(x)}{\xi(x) h(x)-g(x)}=\frac{\alpha \lambda^{2}(1+x)}{1+\lambda(1+x)}, \quad x>0
$$

The general solution of the differential equation in Corollary 4.1 is

$$
\xi(x)=(1+\lambda(1+x))^{-\alpha} e^{\alpha \lambda x}\left[-\int \alpha \lambda^{2}(1+x)(1+\lambda(1+x))^{\alpha-1} e^{-\alpha \lambda x}(h(x))^{-1} g(x)+D\right]
$$

where $D$ is a constant. Note that a set of functions satisfying the above differential equation is given in Proposition 4.1 with $D=0$. However, it should be also noted that there are other triplets $(h, g, \xi)$ satisfying the conditions of Theorem 4.1.

\subsection{Characterization based on hazard function}

It is known that the hazard function, $h_{F}$, of a twice differentiable distribution function, $F$, satisfies the first order differential equation

$$
\frac{f^{\prime}(x)}{f(x)}=\frac{h_{F}^{\prime}(x)}{h_{F}(x)}-h_{F}(x)
$$

For many univariate continuous distributions, this is the only characterization available in terms of the hazard function. The following characterization establish a non-trivial characterization of EGL distribution, for $\beta=1$, in terms of the hazard function, which is not of the above trivial form.

Proposition 4.2 Let $X: \Omega \rightarrow(0, \infty)$ be a continuous random variable. For $\beta=1$, the PDF of $X$ is (2.6) if and only if its hazard function $h_{F}(x)$ satisfies the differential equation

$$
h_{F}^{\prime}(x)-(1+x)^{-1} h_{F}(x)=-\frac{\alpha \lambda^{3}}{(1+\lambda(1+x))^{2}},
$$

with the initial condition $h_{F}(0)=\frac{\alpha \lambda^{2}}{\lambda+1}$.

Proof. If $X$ has PDF (2.6), then clearly the above differential equation holds. Now, it the differential equation holds, then

or

$$
\frac{d}{d x}\left\{(1+x)^{-1} h_{F}(x)\right\}=\alpha \lambda^{2} \frac{d}{d x}\left\{(1+\lambda(1+x))^{-1}\right\}
$$

$$
h_{F}(x)=\frac{\alpha \lambda^{2}(1+x)}{1+\lambda(1+x)}
$$

which is the hazard function of the EGL distribution for $\beta=1$. 


\subsection{Characterization in terms of the reverse (or reversed) hazard function}

The reverse hazard function, $r_{F}$, of a twice differentiable distribution function, $F$, is defined as

$$
r_{F}(x)=\frac{f(x)}{F(x)}, \quad x \in \text { support of } F .
$$

Proposition 4.3 Let $X: \Omega \rightarrow(0, \infty)$ be a continuous random variable. The PDF of $X$ is $(2.6)$ if and only if its reverse hazard function $r_{F}(x)$ satisfies the differential equation

$$
r_{F}^{\prime}(x)-(1+x)^{-1} r_{F}(x)=\frac{\alpha \beta \lambda^{2}}{\lambda+1}(1+x) \frac{d}{d x}\left\{\frac{\left(1+\frac{\lambda x}{\lambda+1}\right)^{\alpha-1}}{1-\left(1+\frac{\lambda x}{\lambda+1}\right)^{\alpha} e^{-\alpha \lambda x}}\right\} .
$$

Proof. If $X$ has PDF (2.6), then clearly the above differential equation holds. Now, if the differential equation holds, then

or

$$
\frac{d}{d x}\left\{(1+x)^{-1} r_{F}(x)\right\}=\frac{\alpha \beta \lambda^{2}}{\lambda+1} \frac{d}{d x}\left\{\frac{\left(1+\frac{\lambda x}{\lambda+1}\right)^{\alpha-1}}{1-\left(1+\frac{\lambda x}{\lambda+1}\right)^{\alpha} e^{-\alpha \lambda x}}\right\},
$$

$$
r_{F}(x)=\frac{\alpha \beta \lambda^{2}(1+x)\left(1+\frac{\lambda x}{\lambda+1}\right)^{\alpha-1}}{(\lambda+1)\left[1-\left(1+\frac{\lambda x}{\lambda+1}\right)^{\alpha} e^{-\alpha \lambda x}\right]},
$$

which is the reverse hazard function of the EGL distribution.

\subsection{Characterizations based on conditional expectation}

The following propositions have already appeared in [16], so we will just state them here which can be used to characterize the EGL distribution.

Proposition 4.4 Let $X: \Omega \rightarrow(a, b)$ be a continuous random variable with $c d f F$. Let $\psi(x)$ be a differentiable function on $(a, b)$ with $\lim _{x \rightarrow a^{+}} \psi(x)=1$. Then for $\delta \neq 1$,

$$
E[\psi(X) \mid X \geq x]=\delta \psi(x), \quad x \in(a, b),
$$

if and only if

$$
\psi(x)=(1-F(x))^{\frac{1}{\delta}-1}, \quad x \in(a, b) .
$$

Proposition 4.5 Let $X: \Omega \rightarrow(a, b)$ be a continuous random variable with $c d f F$. Let $\psi_{1}(x)$ be a differentiable function on $(a, b)$ with $\lim _{x \rightarrow b} \psi_{1}(x)=1$. Then for $\delta_{1} \neq 1$,

$$
E\left[\psi_{1}(X) \mid X \leq x\right]=\delta_{1} \psi_{1}(x), \quad x \in(a, b),
$$


if and only if

$$
\psi_{1}(x)=(F(x))^{\frac{1}{\delta_{1}}-1}, \quad x \in(a, b)
$$

Remarks 4.4 (a) For $\psi(x)=\left(1+\frac{\lambda x}{\lambda+1}\right) e^{-\lambda x}, \beta=1, \delta=\frac{\alpha}{1+\alpha}$ and $(a, b)=(0, \infty)$, Proposition 4.4 provides a characterization of EGL distribution. (b) For $\psi_{1}(x)=1-\left(1+\frac{\lambda x}{\lambda+1}\right)^{\alpha} e^{-\alpha \lambda x}, \delta_{1}=$ $\frac{\beta}{1+\beta}$ and $(a, b)=(0, \infty)$, Proposition 4.5 provides a characterization of EGL distribution. (c) Of course there are other suitable functions than the ones we mentioned above, which are chosen for simplicity.

\section{$5 \quad$ Estimation of Model Parameters}

In this section, we consider the estimation of the three parameters by the methods of moments and maximum likelihood. Suppose $x_{1}, \ldots, x_{n}$ is a random sample of size $n$ from the EGL distribution given by (2.5). Under the method of moments, equating the theoretical moments $E\left(X^{k}\right)$ with the corresponding sample moments,

$$
M_{k}=\frac{1}{n} \sum_{l=1}^{n} x_{l}^{k}, \quad k=1,2,3 .
$$

respectively, one obtains the system of equations

$$
\begin{aligned}
M_{k} & =\alpha \beta \lambda k !\left(\frac{\lambda+1}{\lambda}\right)^{k} \sum_{j=0}^{\infty} \frac{(-1)^{j} \Gamma(\beta)}{\Gamma(\beta-j) j !}\{\Psi(k+1, k+\alpha(j+1)+1 ; \alpha(j+1)(\lambda+1)) \\
& +[(k+1)(\lambda+1) / \lambda] \Psi(k+2, k+\alpha(j+1)+2 ; \alpha(j+1)(\lambda+1))\}
\end{aligned}
$$

which can be solved simultaneously to give estimates for $\alpha, \beta$ and $\lambda$.

Now consider estimation by the method of maximum likelihood. The log-likelihood for a random sample $x_{1}, \ldots, x_{n}$ from the EGL distribution is

$$
\begin{array}{r}
\log L(\alpha, \beta, \lambda)=n \log \alpha+n \log \beta+2 n \log \lambda-n \log (\lambda+1)+\sum_{i=1}^{n} \log \left(1+x_{i}\right)-\alpha \lambda \sum_{i=1}^{n} x_{i} \\
+(\alpha-1) \sum_{i=1}^{n} \log \left(\frac{\lambda+1+\lambda x_{i}}{\lambda+1}\right)+(\beta-1) \sum_{i=1}^{n} \log \left[1-\left(\frac{\lambda+1+\lambda x_{i}}{\lambda+1} e^{-\lambda x_{i}}\right)^{\alpha}\right]
\end{array}
$$

Differentiating the log-likelihood with respect $\alpha, \beta$ and $\lambda$, respectively, and setting the result equal to zero, we obtain the maximum likelihood estimates $\widehat{\alpha}, \widehat{\beta}$ and $\widehat{\lambda}$ of the unknown parameters $\alpha, \beta$ and $\lambda$, respectively.

\section{Application}

Now, consider the parameters estimation by beta-Dagum, beta-Lindley and Lindley distributions. The PDF of beta-Dagum and beta-Lindley distributions are

1. Beta-Dagum (BD):

$$
f(x ; \alpha, \beta, \lambda, a, b)=\frac{a b \lambda}{\mathrm{B}(\alpha, \beta)} x^{-b-1}\left(1+\lambda x^{-b}\right)^{-a \alpha-1}\left[1-\left(1+\lambda x^{-b}\right)^{-a}\right]^{\beta-1}
$$


2. Beta-Lindley (BL), defined by [17]:

$$
f(x ; \alpha, \beta, \lambda)=\frac{\lambda^{2}(1+x) e^{-\beta \lambda x}}{(\lambda+1) \mathrm{B}(\alpha, \beta)}\left(\frac{\lambda+1+\lambda x}{\lambda+1}\right)^{\beta-1}\left(1-\frac{\lambda+1+\lambda x}{\lambda+1} e^{-\lambda x}\right)^{\alpha-1},
$$

where $x>0, \alpha>0, \beta>0, \lambda>0, a>0, b>0$ and $\mathrm{B}(\cdot, \cdot)$ is the beta function defined by

$$
\mathrm{B}(a, b)=\int_{0}^{1} t^{a-1}(1-t)^{b-1} d t .
$$

The data set represents the failure times of the air conditioning system of an airplane. This data set was taken from [18].

The maximum likelihood estimates (MLEs) of the parameters and the values of the Akaike Information Criterion (AIC) are reported in Table 1. The results show that the EGL distribution provides a significantly better fit than the other three models.

Table 1. The maximum likelihood estimates and AIC of the models

\begin{tabular}{llc}
\hline Distribution & \multicolumn{1}{c}{ Maximum Likelihood Estimates } & AIC \\
\hline BD & $\widehat{\alpha}=2.97, \widehat{\beta}=17.91, \widehat{\lambda}=4.68, \widehat{a}=2.20, \widehat{b}=0.33$ & 312.93 \\
BL & $\widehat{\alpha}=0.45, \widehat{\beta}=0.52, \widehat{\lambda}=0.03$ & 311.46 \\
EGL & $\widehat{\alpha}=0.05, \widehat{\beta}=0.64, \widehat{\lambda}=0.28$ & 309.86 \\
Lindley & $\widehat{\lambda}=0.03$ & 321.27 \\
\hline
\end{tabular}

Plots of the estimated PDF of the BD, BL, EGL and Lindley models fitted to these data are given in Fig 2. The figure indicates that the EGL distribution is superior to the other distributions in terms of model fitting.

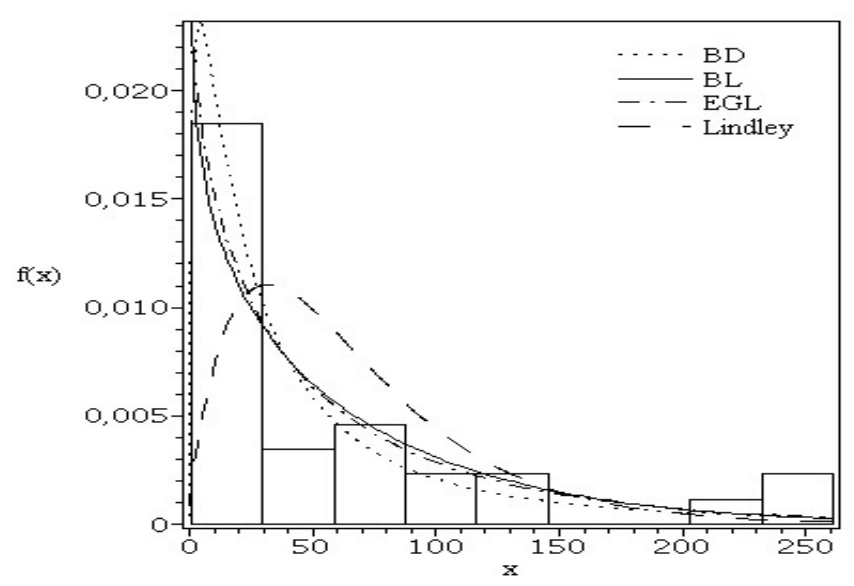

Fig. 2. Histogram and estimated densities

The probability plots consists of plots of the observed probabilities, against the probabilities predicted by the fitted model. Fig. 3 display the probability plots and supports the results shown in Table 1. 


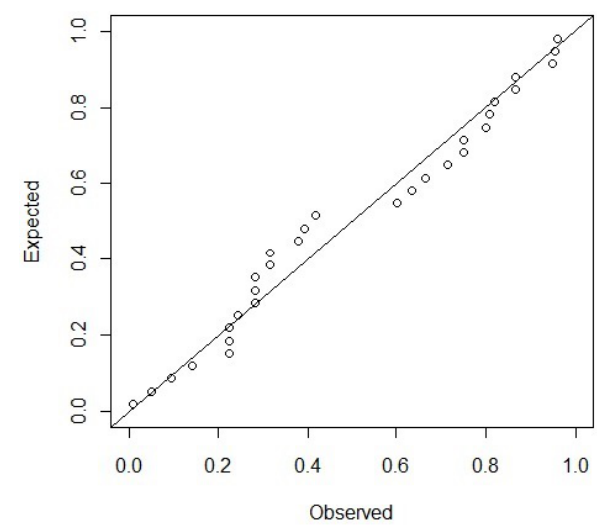

(a) BD distribution.

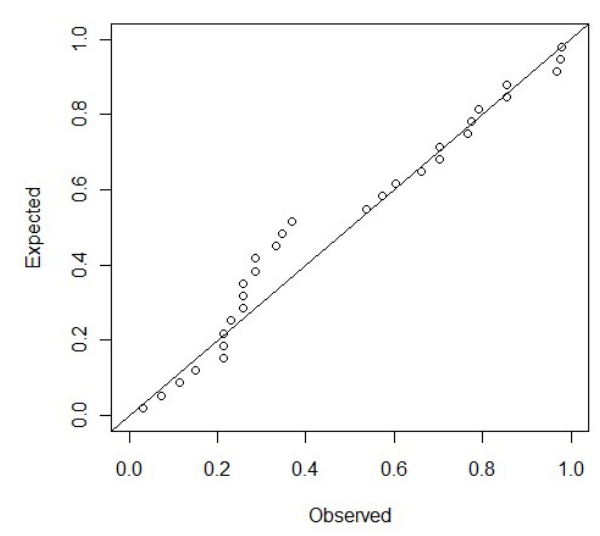

(c) EGL distribution.

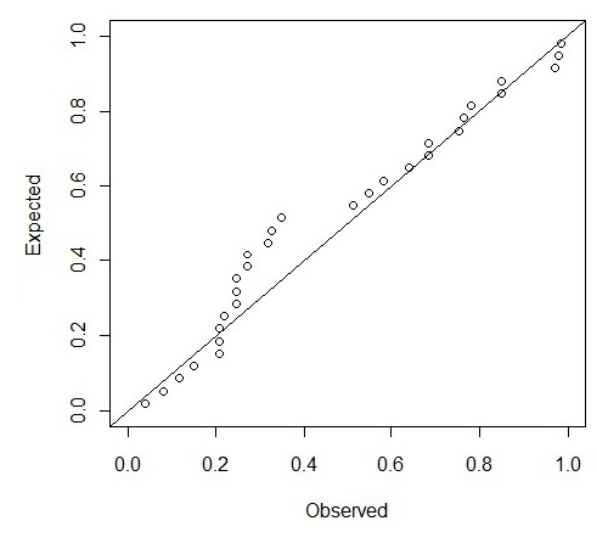

(b) Beta Lindley distribution.

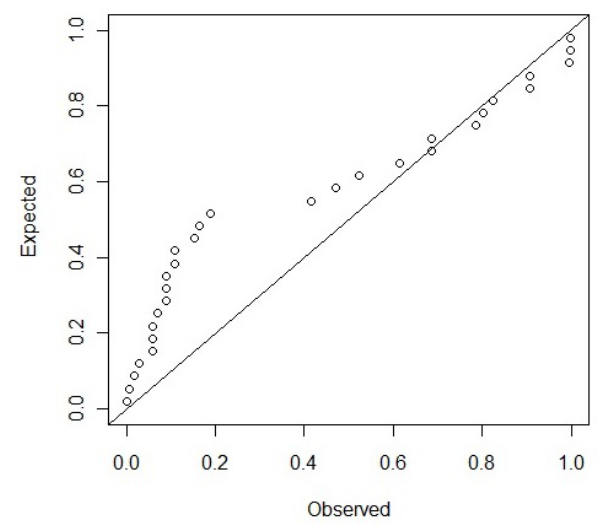

(d) Lindley distribution.

Fig. 3. Probability plots from the fitted distributions

\section{Conclusion}

We proposed a new distribution, named the exponentiated generalized Lindley distribution distribution which extends the Lindley and exponentiated Lindley distributions, among others. Several properties of the new distribution were investigated, including the moments. The estimation of parameters by the method of moments and the maximum likelihood have been discussed. An application of the exponentiated generalized Lindley distribution to a real data show that the new distribution can be used quite effectively to provide better fits than the beta Dagum, beta lindley and Lindley distributions. 


\section{Competing Interests}

Authors have declared that no competing interests exist.

\section{References}

[1] Alzaatreh A, Famoye F, Lee C. Weibull-Pareto distribution and its applications. Communication in Statistics - Theory and Methods. 2013;42(9):1673-1691.

[2] Condino F, Domma F. The Beta-Dagum distribution. Communication in Statistics - Theory and Methods. 2013;42(22):4070-4090.

[3] Oluyede BO, Rajasooriya S. The Mc-Dagum distribution and its statistical properties with applications. Asian Journal of Mathematics and Applications. 2013;2013:1-16.

[4] Nadarajah S, Bakouch HS, Tahmasbi R. A generalized Lindley distribution. Sankhya B. 2011;73:331-359.

[5] Paranaba PF, Ortega EMM, Cordeiro GM, Pescim RR. The beta Burr XII distribution with application to lifetime data. Communication in Statistics - Theory and Methods. 2011;55(2):1118-1136.

[6] Pascoa MAR, Ortega EMM, Cordeiro GM. The Kumaraswamy generalized gamma distribution with application in survival analysis. Communication in Statistics - Theory and Methods. 2011;8(5):411-433.

[7] Pinho LGB, Cordeiro GM, Nobre JS. The gamma-exponentiated Weibull distribution. Journal of Statistical Theory and Applications. 2012;11(4):379-395.

[8] Silva GO, Ortega EMM, Cordeiro GM. The beta modified Weibull distribution. Mathematic and Statistic. 2010;16(3):409-430.

[9] Lindley DV. Fiducial distributions and Bayes' theorem. Journal of the Royal Statistical Society B. 1958;20:102-107.

[10] Cordeiro GM, Ortega EM, Cunha DCC. The exponentiated generalized class of distributions. Journal of Data Science. 2013;11:1-27.

[11] Gradshteyn IS, Ryzhik IM. Table of Integrals, Series, and Products. San Diego: Elsevier; 2007.

[12] Prudnikov AP, Brychkov YA, Marichev OI. Integrals and series, vol.1. Amsterdam: Gordon and Breach; 1998.

[13] Arnold BC, Balakrishnan AN, Nagaraja HN. A first course in order statistics. New York: Wiley-Interscience; 1992.

[14] Glänzel W. A characterization theorem based on truncated moments and its application to some distribution families. Mathematical Statistics and Probability Theory (Bad Tatzmannsdorf, 1986). 1987;B:75-84.

[15] Glänzel W. Some consequences of a characterization theorem based on truncated moments. Statistics: A Journal of Theoretical and Applied Statistics. 1990;21(4):613-618. 
[16] Hamedani GG. On certain generalized gamma convolution distributions II. Technical Report - Marquette University. 2013;484.

[17] Merovci F, Sharma VK. The beta Lindley distribution: Properties and applications. Journal of Applied Mathematics. 2014;2014:1-10.

[18] Linhart H, Zucchini W. Model selection. New York: Wiley; 1986.

(C) 2017 Rodrigues et al.; This is an Open Access article distributed under the terms of the Creative Commons Attribution License (http://creativecommons.org/licenses/by/4.0), which permits unrestricted use, distribution, and reproduction in any medium, provided the original work is properly cited.

\section{Peer-review history:}

The peer review history for this paper can be accessed here (Please copy paste the total link in your browser address bar)

http://sciencedomain.org/review-history/20136 